\title{
Optimized selection of three major EGFR-TKIs in advanced EGFR-positive non-small cell lung cancer: a network meta- analysis
}

\author{
Yaxiong Zhang ${ }^{1,2,3, *}$, Jin Sheng ${ }^{1,2,3, *}$, Yunpeng Yang ${ }^{1,2,3, *}$, Wenfeng Fang ${ }^{1,2,3}$, Shiyang \\ Kang $^{2,3,4}$, Yang $\mathrm{He}^{5}$, Shaodong Hong ${ }^{1,2,3}$, Jianhua Zhan ${ }^{1,2,3}$, Yuanyuan Zhao ${ }^{1,2,3}$, \\ Cong Xue ${ }^{1,2,3}$, Yuxiang $\mathrm{Ma}^{1,2,3}$, Ting Zhou ${ }^{1,2,3}$, Shuxiang $\mathrm{Ma}^{1,2,3}$, Fangfang $\mathbf{G a O}^{1,2,3}$, \\ Tao Qin ${ }^{1,2,3}$, Zhihuang Hu${ }^{1,2,3}$, Ying Tian ${ }^{1,2,3}$, Xue Hou ${ }^{1,2,3}$, Yan Huang ${ }^{1,2,3}$, Ningning \\ Zhou $^{1,2,3}$, Hongyun Zhao ${ }^{1,2,3}$, Li Zhang ${ }^{1,2,3}$ \\ ${ }^{1}$ Department of Medical Oncology, Sun Yat-sen University Cancer Center, Guangzhou, China \\ ${ }^{2}$ State Key Laboratory of Oncology in South China, Guangzhou, China \\ ${ }^{3}$ Collaborative Innovation Center for Cancer Medicine, Guangzhou, China \\ ${ }^{4}$ Department of Anesthesiology, Sun Yat-sen University Cancer Center, Guangzhou, China \\ ${ }^{5}$ Lau Luen Hung Private Medical Center, the First Affiliated Hospital of Sun Yat-sen University, Guangzhou, China \\ *These authors have contributed equally to this work \\ Correspondence to: Li Zhang, e-mail: zhangli6@mail.sysu.edu.cn \\ Keywords: EGFR-TKI, NSCLC, gefitinib, erlotinib, afatinib \\ Received: January 21, $2016 \quad$ Accepted: February 18, $2016 \quad$ Published: February 25, 2016
}

\section{ABSTRACT}

Background: To answer which epidermal growth factor receptor-tyrosine kinase inhibitor (EGFR-TKI) is the best choice for advanced non-small cell lung cancer (NSCLC) EGFR mutants.

Results: 16 phase III randomized trials involving 2962 advanced NSCLC EGFR mutants were enrolled. Multiple treatment comparisons showed different EGFRTKIs shared equivalent curative effect in terms of all outcome measures among the overall, chemo-naïve and previously treated patients. Rank probabilities showed that erlotinib and afatinib had potentially better efficacy compared with gefitinib in both of the overall and chemo-naïve patients. Potentially survival benefit of erlotinib was also observed in previously treated patients compared with gefitinib. Additionally, EGFR-TKI showed numerically greater survival benefit in 19 Del compared with chemotherapy, while it was opposite in 21 L858R. Furthermore, afatinib, erlotinib and gefitinib had high, moderate and low risk of rash \& diarrhea, respectively, while the occurrence of elevated liver transaminase was more common in gefitinib.

Methods: Data of objective response rate (ORR), disease control rate (DCR), progression-free survival (PFS), overall survival (OS) and adverse events were extracted from included studies. Efficacy and toxicity of all included treatments were integrated by network meta-analyses.

Conclusion: Our study indicated a high efficacy-high toxicity pattern of afatinib, a high efficacy-moderate toxicity pattern of erlotinib and a medium efficacy-moderate toxicity pattern of gefitinib. Recommended EGFR-TKI should be suggested according to patients' tolerability and therapeutic efficacy in clinical practice. Moreover, the treatment for advanced EGFR-positive NSCLC might be different between $19 \mathrm{Del}$ and 21 L858R. 


\section{INTRODUCTION}

It has been proved that three major epidermal growth factor receptor-tyrosine kinase inhibitors (EGFRTKIs - gefitinib, erlotinib and afatinib) were the best choice for advanced chemo-naïve non-small cell lung cancer (NSCLC) patients harboring sensitive EGFR mutations from nine phase III, randomized trials. [1-9] Interestingly, the combined analyses of LUX-Lung 3 and LUX-Lung 6 showed that only patients with EGFR exon 19 deletions (19 Del) got overall survival benefit from afatinib compared with chemotherapy. By contrast, there were no significant differences between afatinib and chemotherapy in terms of survival for patients with EGFR L858R substitution in exon 21 (21 L858R). [10] After that, more and more studies focused on the subtypes of sensitive EGFR mutations, 19 Del and 21 L858R. Several meta-analyses proved that NSCLC patients with 19 Del had higher response rates and longer progressionfree survival (PFS), overall survival (OS) after EGFRTKI therapy compared with L858R. [11, 12] Moreover, a recent study found that NSCLC patients with 19 Del are more likely to be young and have lymphatic metastasis than those with L858R. [13] These findings suggest that EGFR 19 Del disease might be distinct from 21 L858R disease. Subgroups of 19 Del and L858R should be analyzed separately for therapeutic efficacy and toxicity in future researches.

With those different EGFR-TKIs, scientists performed some head-to-head randomized controlled trials (RCTs) to compare the efficacy and toxicity between gefitinib and erlotinib in chemo-naïve or previously treated patients. However, there was no statistically significant difference in response rates, PFS and OS for EGFR-mutated NSCLC. [14, 15] Additionally, a latest blockbuster trial which directly compared the first generation TKI with second generation TKI found that afatinib significantly improved the response rates and PFS in EGFR-mutant NSCLC versus gefitinib. Subgroup analyses found consistent effects in 19 Del patients and L858R patients. [16]

Up to now, we have sufficient clinical data of EGFRTKIs in EGFR-positive NSCLC patients (EGFR mutants, 19 Del or 21 L858R cases). It is high time for us perform a large-scale analysis to answer which EGFR-TKI is the best clinical choice for EGFR-positive patients, 19 Del patients or L858R patients. Besides, we can analyze whether the optimized selection of EGFR-TKIs is different between chemo-naïve patients and previously treated ones. Since a single trial or conventional direct meta-analysis usually compares only two drugs, it is impossible to integrate information on the relative efficacy and toxicity of all optional regimens for the same indication. Therefore, a network meta-analysis which synthesizes data from both direct and indirect comparisons of diverse regimens is a superexcellent method to compare different treatments due to its good agreement on the real-world situation. [17] This efficacy and toxicity based network meta-analysis will help clinicians make precise choice of EGFR-TKI for advanced NSCLC EGFR mutants.

\section{RESULTS}

\section{Eligible studies}

1124 records were identified according to the primary search strategy and finally 16 phase III randomized trials were enrolled, [1-9, 14-16, 18-21] which involved 2962 advanced NSCLC patients with EGFR mutations. Figure 1 summarizes the flow chart. 11 trials focused on front-line therapy in 2531 treatmentnaive patients, $[1-9,15,16]$ while 6 trials investigated subsequent treatment after failure of chemotherapy in 522 previously treated patients. [18-21] In particular, we took the overall results of CTONG0901 as first-line trial outcomes because most of patients in CTONG0901 trial were chemo-naïve. The previously treated patients in CTONG0901 were incorporated into $\geq 2$ nd-line treatment group separately. [15] Table 1 summarized the basic characteristics of involved studies for this network metaanalysis. Table S1-S3 summarized the data of treatment efficacy (ORR, DCR, 1y-PFS rate, 1y-OS rate and 2y-OS rate) in EGFR mutants, 19 Del patients $(\mathrm{N}=1142)$ and 21 L858R ones $(\mathrm{N}=872)$. Table $\mathrm{S} 4$ enumerated 3 major adverse effects of EGFR-TKIs (rash, diarrhea and elevated LT) from eligible trials.

\section{Single-arm meta-analyses on efficacy and toxicity of EGFR-TKI}

Table 2 calculated the pooled efficacy and toxicity of EGFR-TKIs, gefitinib, erlotinib and afatinib in EGFR mutants or patients with 19 Del or 21 L858R. Only tiny numerical differences were observed among gefitinib, erlotinib and afatinib in terms of therapeutic efficacy in the whole population of EGFR mutants, as well as in chemo-naïve patients (both 19 Del and L858R). However, in previously treated patients, erlotinib showed better

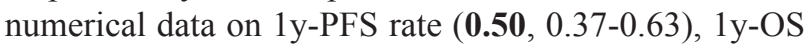
rate $(\mathbf{0 . 7 9}, 0.68-0.87)$ and $2 y-O S$ rate $(\mathbf{0 . 5 7}, 0.37-0.76)$ than gefitinib (1y-PFS, $\mathbf{0 . 3 1}, 0.19-0.46$; $1 \mathrm{y}-\mathrm{OS}$ rate, $\mathbf{0 . 5 9}$, $0.46-0.70 ; 2 y-O S$ rate $\mathbf{0 . 3 0}, 0.20-0.43$ ) (the efficacy of afatinib was not measured in previously treated patients). Particularly, in general, 19 Del patients had superior numerical data on treatment efficacy especially in ORR

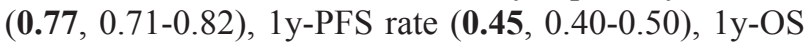
rate $\mathbf{( 0 . 8 7}, 0.83-0.91)$ and $2 \mathrm{y}-\mathrm{OS}$ rate $(\mathbf{0 . 6 2}, 0.57-0.68)$ compared with $21 \mathrm{~L} 858 \mathrm{R}$ patients (ORR, $\mathbf{0 . 6 0}, 0.52-0.67$; $1 \mathrm{y}$-PFS, $\mathbf{0 . 3 8}, 0.33-0.44$; $1 \mathrm{y}-\mathrm{OS}$ rate, $\mathbf{0 . 8 0}, 0.70-0.87 ; 2 \mathrm{y}-$ OS rate $\mathbf{0 . 4 7}, 0.34-0.60)$. Adverse effects were analyzed in treatment-naïve patients. Afatinib showed higher risk of rash $(\mathbf{0 . 8 6}, 0.80-0.91)$ (grade 3-4 rash, $\mathbf{0 . 1 4}, 0.10-0.18$ ) 
and diarrhea (0.91, 0.86-0.95) (grade 3-4 diarrhea, 0.10, 0.06-0.17) than gefitinib or erlotinib, while elevated LT (grade 3-4 elevated LT) was easily occurred in gefitinib $(\mathbf{0 . 3 6}, 0.15-0.65 ; \mathbf{0 . 1 4}, 0.06-0.30)$ compared with afatinib or erlotinib.

\section{Networks for multiple treatment comparisons (MTC)}

Network A and B was established for MTC based on available data of outcomes in EGFR mutants and patients with EGFR 19 Del / 21 L858R, respectively (Figure 2). It was important to note that network $\mathrm{A}$ was composed of first-line trials and $\geq 2$ nd-line trials so that it could be divided into two sub-networks for MTC in chemo-naïve patients or previously treated patients.

\section{Network meta-analyses on therapeutic efficacy in overall \& chemo-naïve EGFR mutants}

According to the data based on network A, gefitinib, elotinib and afatinib shared equivalent curative effect in all outcome measures without significant differences in ORs while all EGFR-TKIs were better than first-line chemotherapy in terms of ORR, DCR and $1 \mathrm{y}$-PFS rate. No significant difference of OS among all TKIs and chemotherapy (Table 3). Analyses of the probability to be the best treatment indicated that afatinib ranked best among all the TKIs in terms of ORR (overall 0.67; chemo- naïve 0.68) and 1-year PFS (overall 0.64; chemo-naïve 0.59 ), while erlotinib ranked best for DCR (overall 0.63; chemo-naïve 0.62). Erlotinib and afatinib shared similar superior rankings with respect to $1 \mathrm{y}-\mathrm{OS}$ (overall 0.41 and 0.39; chemo-naïve 0.39 and 0.46 ) and $2 y-O S$ (overall 0.27 and 0.30 ; chemo-naïve 0.37 and 0.36 ) compared with gefitinib (Figure 3A, 3B, 3G, 3F and Table S5).

\section{Network meta-analyses on therapeutic efficacy in previously treated EGFR mutants}

Only gefitinib, elotinib and chemotherapy did MTC based on sub-network ( $\geq 2$ nd-line trials) of network A. Above three treatments shared equivalent efficacy in all outcome measures by showing no significant differences in ORs (Table 3). Rank probabilities indicated gefitinib ranked best for ORR (0.67) while erlotinib ranked best for $1 y-P F S(0.69), 1 y-O S(0.85)$ and $2 y-O S(0.60)$ compared with gefitinib and $\geq 2$ nd-line chemotherapy (Figure 3C, $3 \mathrm{H}$ and Table S5). DCRs were not evaluated in previously treated EGFR mutants due to not available data.

\section{Network meta-analyses on therapeutic efficacy in patients with EGFR 19 Del / 21 L858R}

According to the data based on network B, gefitinib, elotinib and afatinib showed similar curative effect in all outcome measures without significant differences in ORs in EGFR 19 Del patients, while afatinib had better ORR

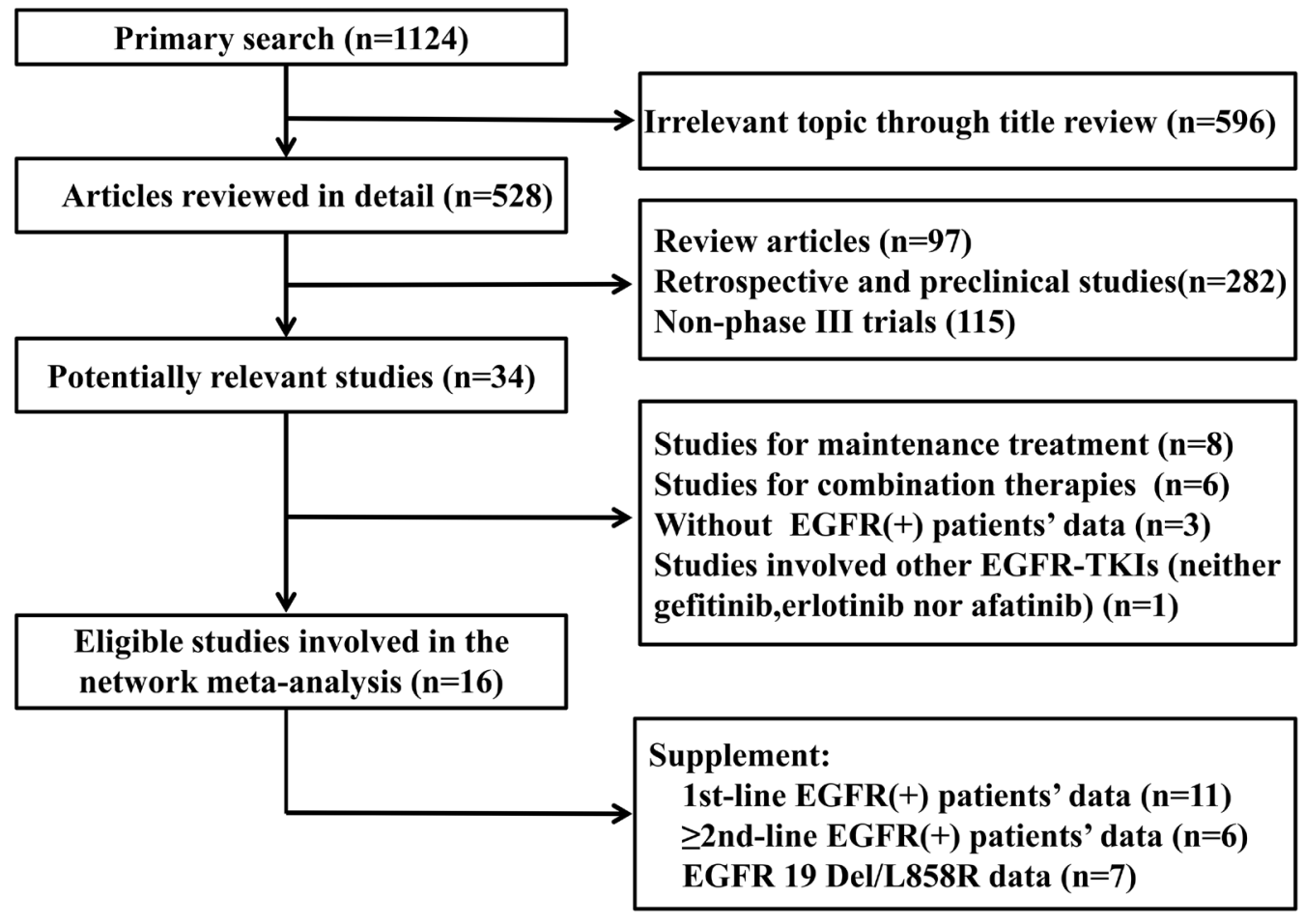

Figure 1: Profile summarizing the trial flow. Abbreviations:19 Del, exon 19 deletion; 21 L858R, exon 21 L858R mutation. 
Table 1: Characteristics of included studies for meta-analyses

\begin{tabular}{|c|c|c|c|c|}
\hline Trial (year) & Type & Race & Drug & EGFR mutants \\
\hline IPASS & CT naive & Asian & Gefitinib & 132 \\
\hline (2009) & & & $\mathrm{TC}$ & 129 \\
\hline NEJ002 & CT naive & Asian & Gefitinib & 114 \\
\hline (2010) & & & $\mathrm{TC}$ & 114 \\
\hline WJTOG3405 & CT naive & Asian & Gefitinib & 86 \\
\hline (2010) & & & DP & 86 \\
\hline OPTIMAL & CT naive & Asian & Erlotinib & 82 \\
\hline (2011) & & & GC & 72 \\
\hline First-SIGNAL & CT naive & Asian & Gefitinib & 26 \\
\hline (2012) & & & $\mathrm{GC}$ & 16 \\
\hline EURTAC & CT naive & Caucasian & Erlotinib & 86 \\
\hline (2012) & & & GP/DP/GC/DC & 87 \\
\hline LUX-Lung 3 & CT naive & Mixed & Afatinib & 230 \\
\hline (2013) & & & $\mathrm{AP}$ & 115 \\
\hline LUX-Lung 6 & CT naive & Asian & Afatinib & 242 \\
\hline (2014) & & & GP & 122 \\
\hline ENSURE & CT naive & Asian & Erlotinib & 110 \\
\hline (2015) & & & GP & 107 \\
\hline LUX-Lung 7 & CT naive & Mixed & Afatinib & 160 \\
\hline (2015) & & & Gefitinib & 159 \\
\hline$V-15-32$ & Previously treated & Asian & Gefitinib & 9 \\
\hline (2008) & & & $\mathrm{DOC}$ & 11 \\
\hline INTEREST & Previously treated & Mixed & Gefitinib & 19 \\
\hline (2008) & & & DOC & 19 \\
\hline TITAN & Previously treated & Mixed & Erlotinib & 7 \\
\hline (2012) & & & PEM/DOC & 4 \\
\hline DELTA & Previously treated & Caucasian & Erlotinib & 21 \\
\hline (2014) & & & $\mathrm{DOC}$ & 30 \\
\hline WJOG 5108L & Previously treated & Asian & Gefitinib & 161 \\
\hline (2014) & & & Erlotinib & 150 \\
\hline CTONG 0901 & $\begin{array}{l}\text { Mixed(mainly on CT } \\
\text { naïve) naïve) }\end{array}$ & Asian & Gefitinib & 128 \\
\hline (2015) & & & Erlotinib & 128 \\
\hline CTONG 0901 & Previously treated & Asian & Gefitinib & 44 \\
\hline (2015) & & & Erlotinib & 47 \\
\hline
\end{tabular}

Abbreviations: TC, carboplatin plus palitaxel; GP, cisplatin plus gemcitabine; DP, cisplatin plus docetaxel; DC, carboplatin plus docetaxel; DOC, docetaxel; GC, carboplatin plus gemcitabine; CT, chemotherapy; PEM, pemetrexed; AP, cisplatin plus pemetrexed; NA, not available. 
Table 2: single-arm meta-analyses on efficacy and toxicity of EGFR-TKIs, gefitinib, erlotinib and afatinib in EGFR mutants or patients with 19 Del / 21 L858R

\begin{tabular}{|c|c|c|c|c|}
\hline Efficacy / Toxicitiy & EGFR-TKIs & Gefitinib & Erlotinib & Afatinib \\
\hline \multicolumn{5}{|l|}{ EGFR mutants (Total) } \\
\hline ORR & $0.65(0.61,0.69)$ & $0.64(0.57,0.70)$ & $0.66(0.57,0.74)$ & $0.66(0.56,0.75)$ \\
\hline DCR & $0.90(0.88,0.92)$ & $0.88(0.85,0.91)$ & $0.90(0.86,0.92)$ & $0.94(0.89,0.96)$ \\
\hline 1y-PFS & $0.44(0.40,0.49)$ & $0.40(0.36,0.43)$ & $0.47(0.37,0.57)$ & $0.52(0.47,0.57)$ \\
\hline $1 \mathrm{y}-\mathrm{OS}$ & $0.80(0.77,0.83)$ & $0.78(0.71,0.84)$ & $0.80(0.75,0.85)$ & $0.82(0.78,0.85)$ \\
\hline $2 y-O S$ & $0.51(0.46,0.56)$ & $0.50(0.40,0.60)$ & $0.50(0.44,0.57)$ & $0.52(0.38,0.65)$ \\
\hline \multicolumn{5}{|l|}{ EGFR mutants (First-line) } \\
\hline ORR & $0.66(0.61,0.71)$ & $0.66(0.57,0.74)$ & $0.68(0.58,0.77)$ & $0.66(0.56,0.75)$ \\
\hline DCR & $0.91(0.88,0.93)$ & $0.89(0.85,0.92)$ & $0.90(0.85,0.94)$ & $0.94(0.89,0.96)$ \\
\hline 1y-PFS & $0.45(0.40,0.50)$ & $0.40(0.36,0.44)$ & $0.47(0.34,0.61)$ & $0.52(0.47,0.57)$ \\
\hline $1 \mathrm{y}-\mathrm{OS}$ & $0.80(0.78,0.83)$ & $0.80(0.74,0.85)$ & $0.80(0.74,0.85)$ & $0.82(0.78,0.85)$ \\
\hline $2 y-O S$ & $0.51(0.46,0.56)$ & $0.53(0.43,0.62)$ & $0.49(0.44,0.54)$ & $0.52(0.38,0.65)$ \\
\hline \multicolumn{5}{|c|}{ EGFR mutants (After first-line) } \\
\hline ORR & $0.60(0.53,0.66)$ & $0.59(0.45,0.72)$ & $0.58(0.50,0.66)$ & \\
\hline DCR & $0.87(0.83,0.91)$ & $0.86(0.79,0.91)$ & $0.89(0.82,0.93)$ & \\
\hline 1y-PFS & $0.41(0.32,0.51)$ & $0.31(0.19,0.46)$ & $0.50(0.37,0.63)$ & \\
\hline 1y-OS & $0.70(0.57,0.81)$ & $0.59(0.46,0.70)$ & $0.79(0.68,0.87)$ & \\
\hline $2 y-O S$ & $0.45(0.29,0.62)$ & $0.30(0.20,0.43)$ & $0.57(0.37,0.76)$ & \\
\hline \multicolumn{5}{|l|}{ EGFR 19 Del (First-line) } \\
\hline ORR & $0.77(0.71,0.82)$ & $0.81(0.72,0.87)$ & $0.78(0.64,0.89)$ & $0.74(0.64,0.83)$ \\
\hline DCR & $0.96(0.91,0.98)$ & $1.00(0.96,1.00)$ & $0.98(0.88,1.00)$ & $0.94(0.88,0.97)$ \\
\hline 1y-PFS & $0.45(0.40,0.50)$ & $0.42(0.35,0.49)$ & $0.46(0.37,0.55)$ & $0.51(0.40,0.61)$ \\
\hline $1 \mathrm{y}-\mathrm{OS}$ & $0.87(0.83,0.91)$ & NA & $0.86(0.74,0.94)$ & $0.88(0.83,0.91)$ \\
\hline $2 y-O S$ & $0.62(0.57,0.68)$ & NA & $0.60(0.46,0.72)$ & $0.63(0.56,0.70)$ \\
\hline \multicolumn{5}{|l|}{ EGFR 21 L858R (First-line) } \\
\hline ORR & $0.60(0.52,0.67)$ & $0.56(0.46,0.65)$ & $0.73(0.50,0.89)$ & $0.60(0.48,0.72)$ \\
\hline DCR & $0.93(0.89,0.95)$ & $0.94(0.85,0.98)$ & $0.95(0.77,1.00)$ & $0.93(0.86,0.97)$ \\
\hline 1y-PFS & $0.38(0.33,0.44)$ & $0.39(0.32,0.47)$ & $0.32(0.23,0.43)$ & $0.42(0.30,0.54)$ \\
\hline $1 \mathrm{y}-\mathrm{OS}$ & $0.80(0.70,0.87)$ & NA & $0.83(0.70,0.92)$ & $0.79(0.63,0.89)$ \\
\hline $2 y-O S$ & $0.47(0.34,0.60)$ & NA & $0.52(0.38,0.66)$ & $0.45(0.26,0.65)$ \\
\hline \multicolumn{5}{|l|}{ EGFR mutants (First-line) } \\
\hline Rash & $0.78(0.73,0.83)$ & $0.75(0.64,0.84)$ & $0.73(0.68,0.77)$ & $0.86(0.80,0.91)$ \\
\hline Diarrhea & $0.57(0.37,0.75)$ & $0.41(0.23,0.61)$ & $0.35(0.19,0.55)$ & $0.91(0.86,0.95)$ \\
\hline Elevated LT & $0.20(0.11,0.34)$ & $0.36(0.15,0.65)$ & $0.12(0.04,0.31)$ & $0.14(0.06,0.28)$ \\
\hline Grade 3-4 Rash & $0.06(0.04,0.10)$ & $0.03(0.02,0.06)$ & $0.05(0.02,0.12)$ & $0.14(0.10,0.18)$ \\
\hline Grade 3-4 Diarrhea & $0.04(0.02,0.07)$ & $0.01(0.00,0.02)$ & $0.02(0.00,0.10)$ & $0.10(0.06,0.17)$ \\
\hline Grade 3-4 Elevated LT & $0.04(0.02,0.10)$ & $0.14(0.06,0.30)$ & $0.02(0.01,0.06)$ & $0.01(0.00,0.04)$ \\
\hline
\end{tabular}

Abbreviations:19 Del, exon 19 deletion; 21 L858R, exon 21 L858R mutation; ORR, objective response rate; DCR, disease control rate; PFS, progression-free survival; OS, overall survival; LT, liver transaminase, NA, not available. 

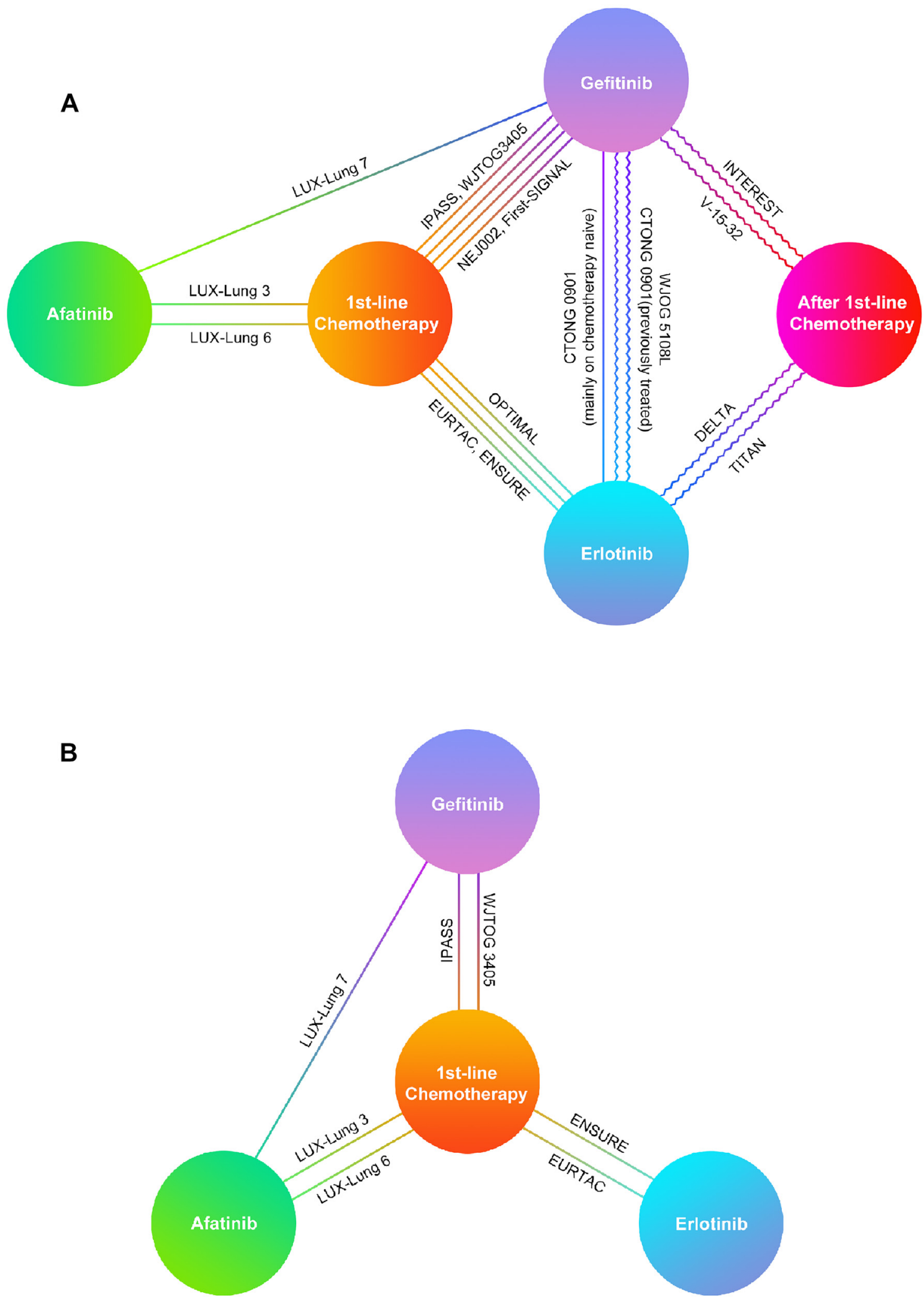

Figure 2: Network established for multiple treatment comparisons. A. For EGFR mutants; B. For patients with EGFR 19 Del or $21 \mathrm{~L} 858 \mathrm{R}$. Straight lines between drugs represented comparisons in chemotherapy-naïve patients. Curve lines between drugs represented comparisons in previously treated patients. Abbreviations:19 Del, exon 19 deletion; 21 L858R, exon 21 L858R mutation. 
Table 3: Multiple treatment comparison for efficacy and toxicities based on network A and network B

\section{Total ORR (EGFR mutants) \\ 1st-line Chemotherapy \\ $0.16(0.09,0.28)$ \\ $0.64(0.16,2.69)$ \\ $0.20(0.11,0.31)$ \\ $0.24(0.15,0.36)$ \\ Total DCR (EGFR mutants)

$\begin{array}{cc}\text { 1st-line Chemotherapy } & 2.36(1.30,4.21) \\ 0.42(0.24,0.77) & \text { Afatinib } \\ 0.36(0.22,0.64) & 0.88(0.42,1.94) \\ 0.55(0.34,0.89) & 1.28(0.68,2.66)\end{array}$

Total 1y-PFS (EGFR mutants)

$\begin{array}{cc}\text { 1st-line Chemotherapy } & 8.33(3.73,20.48) \\ 0.12(0.05,0.27) & \text { Afatinib } \\ 0.50(0.11,2.69) & 4.22(0.80,27.13) \\ 0.16(0.08,0.32) & 1.30(0.48,3.90) \\ 0.16(0.08,0.30) & 1.35(0.54,3.41)\end{array}$

Total 1y-OS (EGFR mutants)

$\begin{array}{cc}\text { 1st-line Chemotherapy } & 1.08(0.64,1.91) \\ 0.92(0.52,1.57) & \text { Afatinib } \\ 2.08(0.65,5.91) & 2.28(0.66,7.52) \\ 0.93(0.59,1.46) & 0.98(0.50,2.07) \\ 1.19(0.78,1.88) & 1.30(0.66,2.70)\end{array}$

Total 2y-OS (EGFR mutants)

$\begin{array}{cc}\begin{array}{c}\text { 1st-line Chemotherapy } \\ 0.97(0.66,1.45)\end{array} & 1.03(0.69,1.52) \\ 1.15(0.46,3.04) & \text { Afatinib } \\ 0.96(0.70,1.36) & 0.98(0.60,1.67) \\ 1.10(0.81,1.51) & 1.12(0.68,1.85)\end{array}$

1st-line ORR (EGFR mutants)

$\begin{array}{cc}\text { 1st-line Chemotherapy } & 6.08(3.47,11.25) \\ 0.16(0.09,0.29) & \text { Afatinib } \\ 0.19(0.11,0.31) & 1.19(0.55,2.41) \\ 0.24(0.14,0.36) & 1.44(0.74,2.68)\end{array}$

$1.55(0.37,6.10)$

$0.26(0.06,1.07)$

After 1st-line

Chemotherapy

$0.31(0.07,1.22)$

$0.37(0.10,1.31)$

$2.75(1.56,4.52)$

$1.13(0.52,2.39)$

Erlotinib

$1.46(0.89,2.46)$

$2.00(0.37,9.12)$

$0.24(0.04,1.25)$

After 1st-line

Chemotherapy

$0.31(0.07,1.23)$

$0.32(0.06,1.41)$

$0.48(0.17,1.53)$

$0.44(0.13,1.51)$

After 1st-line

Chemotherapy

$0.44(0.16,1.27)$

$0.58(0.21,1.68)$

$0.87(0.33,2.16)$

$0.84(0.31,2.20)$

After 1st-line

Chemotherapy

$0.84(0.34,2.11)$

$0.95(0.37,2.41)$

$5.15(3.22,8.98)$

$0.84(0.42,1.80)$

Erlotinib

$1.21(0.72,2.02)$
$5.07(3.20,8.74)$

$4.22(2.80,6.69)$

$0.84(0.42,1.75) \quad 0.69(0.38,1.28)$

$3.26(0.82,14.04) \quad 2.71(0.76,10.51)$

Erlotinib

$0.84(0.49,1.35)$

$1.19(0.74,2.05)$

Gefitinib

$1.83(1.12,2.94)$

$0.78(0.38,1.47)$

$0.68(0.41,1.12)$

Gefitinib

$$
\begin{array}{cc}
6.42(3.12,12.86) & 6.19(3.35,12.00) \\
0.77(0.26,2.09) & 0.74(0.29,1.85) \\
3.25(0.81,14.64) & 3.08(0.71,17.42) \\
\text { Erlotinib } & 0.96(0.49,2.14) \\
1.04(0.47,2.05) & \text { Gefitinib }
\end{array}
$$

$1.08(0.69,1.71)$

$0.84(0.53,1.29)$

$1.02(0.48,2.01)$

$0.77(0.37,1.51)$

$2.28(0.79,6.35)$

Erlotinib

$1.31(0.77,2.24)$

$1.72(0.60,4.65)$

$0.76(0.45,1.30)$

Gefitinib

$1.04(0.74,1.43)$

$0.91(0.66,1.24)$

$1.02(0.60,1.66)$

$0.89(0.54,1.46)$

$1.19(0.47,2.95)$

$1.05(0.41,2.70)$

Erlotinib

$0.87(0.62,1.31)$

$1.15(0.76,1.62)$

Gefitinib

$4.25(2.81,7.00)$

$0.70(0.37,1.35)$

$0.83(0.50,1.39)$

Gefitinib 
1st-line DCR (EGFR mutants)

$$
\begin{gathered}
\text { 1st-line Chemotherapy } \\
0.42(0.23,0.79) \\
0.37(0.19,0.65) \\
0.56(0.32,0.93)
\end{gathered}
$$

$2.37(1.27,4.31)$

Afatinib

$0.87(0.35,1.90)$

$1.32(0.65,2.55)$

1st-line 1y-PFS (EGFR mutants)

$$
\begin{array}{cc}
\text { 1st-line Chemotherapy } & 8.31(3.19,23.21) \\
0.12(0.04,0.31) & \text { Afatinib } \\
0.15(0.07,0.37) & 1.28(0.37,4.74) \\
0.16(0.07,0.35) & 1.37(0.48,3.96)
\end{array}
$$

1st-line 1y-OS (EGFR mutants)

$$
\begin{array}{cc}
\text { 1st-line Chemotherapy } & 1.11(0.62,1.96) \\
0.90(0.51,1.61) & \text { Afatinib } \\
0.92(0.58,1.50) & 1.02(0.48,2.17) \\
1.25(0.79,1.99) & 1.39(0.65,2.86)
\end{array}
$$

1st-line 2y-OS (EGFR mutants)

$$
\begin{array}{cc}
\text { 1st-line Chemotherapy } & 1.00(0.70,1.46) \\
1.00(0.68,1.43) & \text { Afatinib } \\
0.98(0.70,1.38) & 0.99(0.61,1.66) \\
1.10(0.81,1.51) & 1.10(0.68,1.82)
\end{array}
$$

After 1st-line ORR (EGFR mutants)

$$
\begin{array}{lc}
\begin{array}{l}
\text { After 1st-line } \\
\text { Chemotherapy }
\end{array} & 2.22(0.36,12.79) \\
0.45(0.08,2.75) & \text { Erlotinib } \\
0.35(0.09,1.42) & 0.77(0.24,2.52)
\end{array}
$$

After 1st-line 1y-PFS (EGFR mutants)

$$
\begin{aligned}
& \begin{array}{l}
\text { After 1st-line } \\
\text { Chemotherapy } \\
0.28(0.04,1.51) \\
0.38(0.04,2.68)
\end{array}
\end{aligned}
$$$$
3.63(0.66,25.65)
$$$$
\text { Erlotinib }
$$$$
1.38(0.32,5.19)
$$

After 1st-line 1y-OS (EGFR mutants)

$$
\begin{aligned}
& \begin{array}{l}
\text { After 1st-line } \\
\text { Chemotherapy } \\
0.38(0.11,1.31) \\
0.70(0.20,2.58)
\end{array}
\end{aligned}
$$

$$
2.65(0.76,9.46)
$$

Erlotinib $1.92(0.62,5.51)$

After 1st-line 2y-OS (EGFR mutants)

$\begin{array}{lc}\begin{array}{l}\text { After 1st-line } \\ \text { Chemotherapy }\end{array} & 1.29(0.34,4.55) \\ 0.78(0.22,2.92) & \text { Erlotinib } \\ 1.38(0.34,5.72) & 1.76(0.48,6.02)\end{array}$

$2.71(1.54,5.14)$

$1.79(1.07,3.12)$

$1.15(0.53,2.82)$

Erlotinib

$1.53(0.80,3.02)$

$6.57(2.74,15.10)$

$6.12(2.87,13.50)$

$0.78(0.21,2.69)$

Erlotinib

$1.07(0.38,2.79)$

$1.08(0.67,1.72)$

$0.98(0.46,2.09)$

Erlotinib

$1.35(0.78,2.37)$

$1.02(0.72,1.43)$

$1.01(0.60,1.64)$

Erlotinib

$1.13(0.75,1.63)$

$2.83(0.71,11.29)$

$1.29(0.40,4.13)$

Gefitinib

$2.61(0.37,27.85)$

$0.72(0.19,3.16)$

Gefitinib

$1.42(0.39,4.88)$

$0.52(0.18,1.62)$

Gefitinib

$0.72(0.17,2.97)$

$0.57(0.17,2.07)$

Gefitinib
$0.76(0.39,1.55)$

$0.65(0.33,1.25)$

Gefitinib

$0.73(0.25,2.09)$

$0.93(0.36,2.65)$

Gefitinib

$0.80(0.50,1.27)$

$0.72(0.35,1.55)$

$0.74(0.42,1.29)$

Gefitinib

$0.91(0.66,1.23)$

$0.91(0.55,1.47)$

$0.89(0.61,1.34)$

Gefitinib 


\section{1st-line ORR (19 Del)}

1st-line Chemotherapy

$0.12(0.04,0.36)$

$0.19(0.03,1.16)$

$0.14(0.04,0.54)$

1st-line DCR (19 Del)

1st-line Chemotherapy

$0.28(0.06,1.28)$

2E3 (0.27, 9E13)

$0.00(0.00,9 \mathrm{E} 12)$

1st-line 1y-PFS (19 Del)

1st-line Chemotherapy

$0.10(0.00,2.25)$

$0.07(0.01,0.45)$

$0.14(0.02,0.90)$

1st-line 1y-OS (19 Del)

1st-line Chemotherapy

$0.51(0.22,1.14)$

$0.85(0.23,3.00)$

1st-line 2y-OS (19 Del)

1st-line Chemotherapy

$0.50(0.23,1.07)$

$0.70(0.23,2.21)$

1st-line ORR (21 L858R)

1st-line Chemotherapy

$0.22(0.09,0.53)$

$0.44(0.08,2.13)$

$0.67(0.22,1.92)$

1st-line DCR (21 L858R)

1st-line Chemotherapy

$0.28(0.06,1.22)$

$5 \mathrm{E} 5(0.85,2 \mathrm{E} 18)$

$1.48(0.06,85.73)$

1st-line 1y-PFS (21 L858R)

1st-line Chemotherapy

$0.20(0.02,1.50)$

$0.20(0.05,0.81)$

$0.20(0.05,0.71)$
$8.32(2.76,25.95)$

Afatinib

$1.53(0.18,13.17)$

$1.12(0.29,4.48)$

$3.53(0.78,15.40)$

Afatinib

9E3 $(0.79,3 \mathrm{E} 14)$

$0.01(0.00,3 \mathrm{E} 13)$

$10.47(0.44,242.61)$

Afatinib

$0.76(0.02,32.80)$

$1.41(0.12,18.20)$

$1.98(0.87,4.52)$

Afatinib

$1.67(0.36,7.35)$

$2.02(0.94,4.28)$

Afatinib

$1.43(0.36,5.74)$

$4.44(1.88,11.36)$

Afatinib

$1.93(0.30,12.24)$

$2.99(1.02,8.69)$

$3.54(0.82,16.07)$

Afatinib

1E6 (2.44, 9E18)

$5.25(0.31,206.14)$

$5.09(0.67,46.45)$

Afatinib

$1.05(0.10,13.86)$

$1.02(0.21,5.40)$
$5.32(0.86,32.59)$

$0.65(0.08,5.44)$

Erlotinib

$0.72(0.07,6.96)$

$0.00(0.00,3.73)$

$0.00(0.00,1.26)$

Erlotinib

$0.00(0.00,6 \mathrm{E} 9)$

$13.76(2.21,112.29)$

$1.32(0.03,58.35)$

Erlotinib

$1.99(0.14,30.48)$

$1.18(0.33,4.31)$

$0.60(0.14,2.79)$

Erlotinib

$1.42(0.45,4.34)$

$0.70(0.17,2.75)$

Erlotinib

$$
\begin{gathered}
2.26(0.47,12.96) \\
0.52(0.08,3.36) \\
\text { Erlotinib }
\end{gathered}
$$

$1.55(0.21,11.13)$

$0.00(0.00,1.18)$

$0.00(0.00,0.41)$

Erlotinib

$0.00(0.00,6.35)$

$4.88(1.24,20.48)$

$0.95(0.07,10.44)$

Erlotinib

$0.99(0.14,6.53)$
$7.01(1.11,46.12)$

$7.37(1.85,27.80)$

$0.89(0.22,3.48)$

$1.39(0.14,13.80)$

Gefitinib

$565.06(0.00,3 \mathrm{E} 16)$

$177.99(0.00,8 \mathrm{E} 15)$

7E6 $(0.00,3 \mathrm{E} 23)$

Gefitinib

$0.71(0.05,8.28)$

$0.50(0.03,7.16)$

Gefitinib

$$
\begin{gathered}
1.49(0.52,4.62) \\
0.33(0.12,0.98) \\
0.65(0.09,4.69) \\
\text { Gefitinib }
\end{gathered}
$$

$0.68(0.01,16.19)$ $0.19(0.00,3.20)$ $3 \mathrm{E} 5(0.16,1 \mathrm{E} 18)$

\section{Gefitinib}

4.88 (1.42, 19.92)

$0.98(0.19,4.74)$

$1.01(0.15,7.17)$

Gefitinib

(Continued) 
1st-line 1y-OS (21 L858R)

1st-line Chemotherapy

$$
\begin{array}{cc}
0.50(0.21,1.14) & 1.15(0.33,4.53) \\
\text { Afatinib } & 2.30(0.52,11.23) \\
0.44(0.09,1.93) & \text { Erlotinib }
\end{array}
$$

$1.98(0.88,4.79)$

$0.87(0.22,3.05)$

1st-line 2y-OS (21 L858R)

1st-line Chemotherapy

$0.50(0.21,1.15)$

$0.76(0.22,2.56)$

$2.01(0.87,4.68)$

Afatinib

$1.32(0.39,4.55)$

$0.66(0.15,2.91)$

$1.52(0.34,6.62)$

Erlotinib

1st-line Rash (EGFR mutants)

$\begin{array}{cc}\text { 1st-line Chemotherapy } & 62.76(15.29,284.27) \\ 0.02(0.00,0.07) & \text { Afatinib } \\ 0.04(0.01,0.12) & 2.23(0.36,15.08) \\ 0.04(0.01,0.15) & 2.57(0.48,13.61)\end{array}$

$27.77(8.24,101.86)$

$24.42(6.74,92.46)$

$$
\begin{gathered}
0.45(0.07,2.81) \\
\text { Erlotinib }
\end{gathered}
$$

$0.39(0.07,2.09)$

$1.15(0.25,5.19)$

$0.87(0.19,4.07)$

\section{Gefitinib}

1st-line Diarrhea (EGFR mutants)

$\begin{array}{cc}\text { 1st-line Chemotherapy } & 61.67(20.69,191.84) \\ 0.02(0.01,0.05) & \text { Afatinib } \\ 0.16(0.06,0.41) & 9.56(2.41,38.61) \\ 0.18(0.06,0.46) & 10.84(3.17,36.09)\end{array}$

$6.44(2.46,17.02)$

$5.67(2.19,15.74)$

$0.10(0.03,0.41)$

$0.09(0.03,0.32)$

Erlotinib

$1.13(0.35,3.64)$

$0.88(0.27,2.88)$

Gefitinib

1st-line Elevated LT (EGFR mutants)

$\begin{array}{cc}\text { 1st-line Chemotherapy } & 1.19(0.43,3.31) \\ 0.84(0.30,2.33) & \text { Afatinib } \\ 0.61(0.27,1.28) & 0.74(0.20,2.40) \\ 0.31(0.14,0.63) & 0.37(0.13,0.97)\end{array}$

1st-line Grade 3-4 Rash (EGFR mutants)

$\begin{array}{cc}\text { 1st-line Chemotherapy } & 207.88(7.29,5 \mathrm{E} 4) \\ 0.00(0.00,0.14) & \text { Afatinib } \\ 0.01(0.00,0.16) & 1.04(0.00,386.86) \\ 0.09(0.00,2.16) & 16.80(0.45,2 \mathrm{E} 3)\end{array}$

1st-line Grade 3-4 Diarrhea (EGFR mutants)

1st-line Chemotherapy 2E6 (224.49, 2E15)

$\begin{array}{cc}0.00(0.00,0.00) & \text { Afatinib } \\ 0.00(0.00,0.01) & 0.00(0.00,1 \mathrm{E} 10) \\ 0.00(0.00,0.08) & 12.77(0.11,1 \mathrm{E} 3)\end{array}$

1st-line Grade 3-4 Elevated LT (EGFR mutants)

$\begin{array}{cc}\text { 1st-line Chemotherapy } & 0.48(0.01,7.22) \\ 2.07(0.14,85.06) & \text { Afatinib } \\ 0.12(0.00,1.88) & 0.05(0.00,2.38) \\ 0.03(0.00,0.23) & 0.01(0.00,0.20)\end{array}$

Abbreviations:19 Del, exon 19 deletion; 21 L858R, exon 21 L858R mutation; ORR, objective response rate; DCR, disease control rate; PFS, progression-free survival; OS, overall survival; LT, liver transaminase.

$3.26(1.59,7.00)$

$2.70(1.03,7.73)$

$2.01(0.76,5.04)$

Gefitinib

$11.01(0.46,806.99)$

$0.06(0.00,2.21)$

$0.06(0.00,4.85)$

Gefitinib

1E5 (12.91, 2E14)

$0.08(0.00,8.85)$

$0.00(0.00,7 \mathrm{E} 8)$

Gefitinib

$5 \mathrm{E} 4(0.00,8 \mathrm{E} 12)$

$8.37(0.53,1 \mathrm{E} 3)$

$38.04(4.38,625.53)$

$18.94(0.42,1 \mathrm{E} 4)$

$84.42(4.94,5 \mathrm{E} 3)$

Erlotinib

$4.38(0.03,182.97)$

Gefitinib 
A

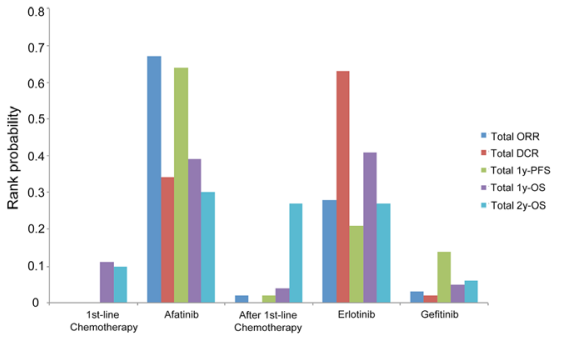

B

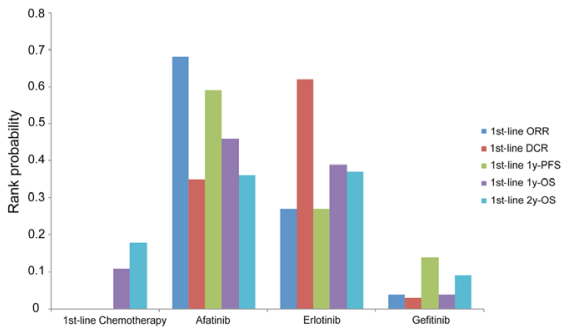

C

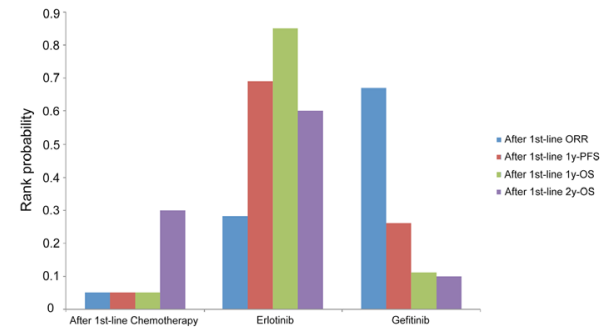

D

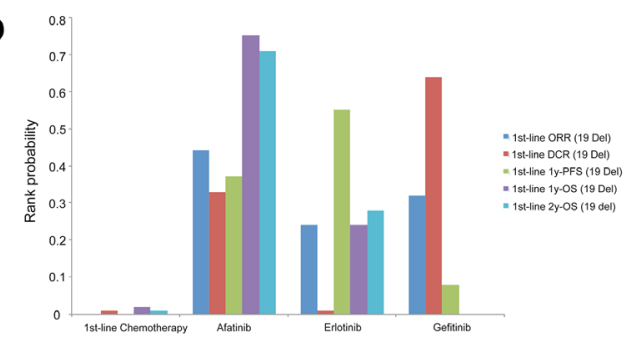

E

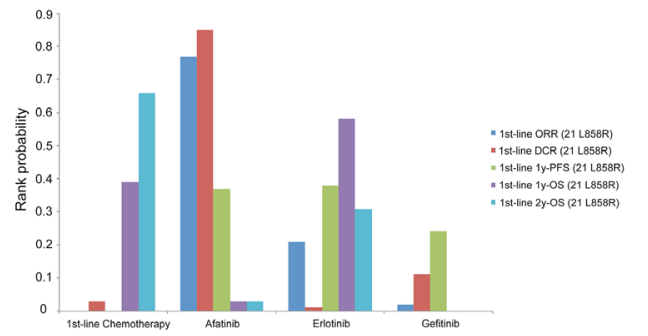

F

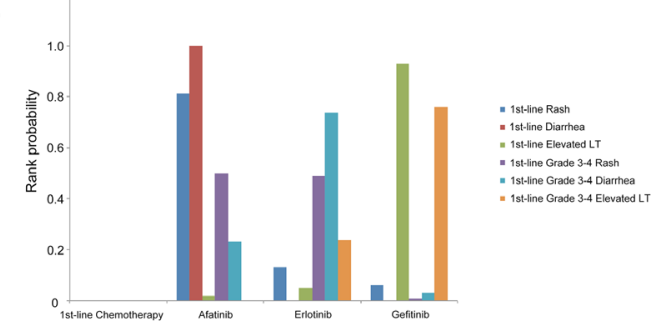

G

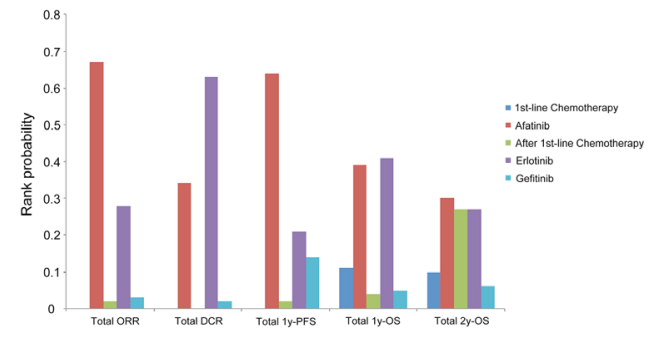

F

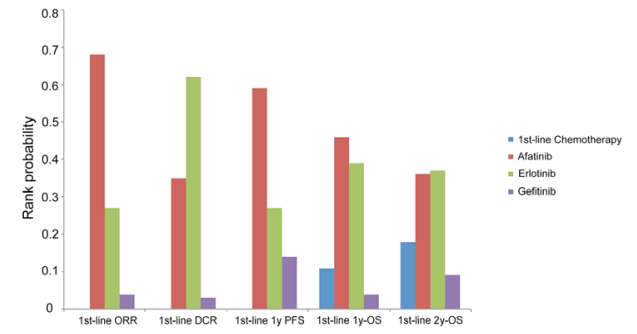

H

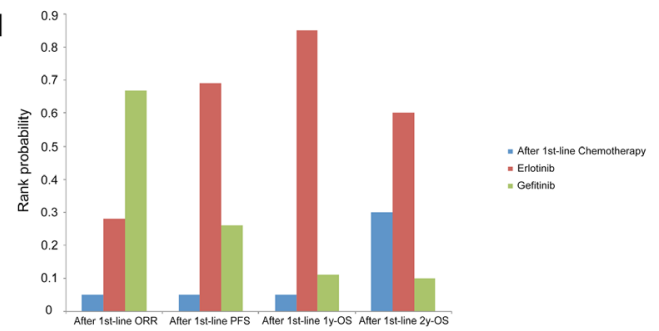

I

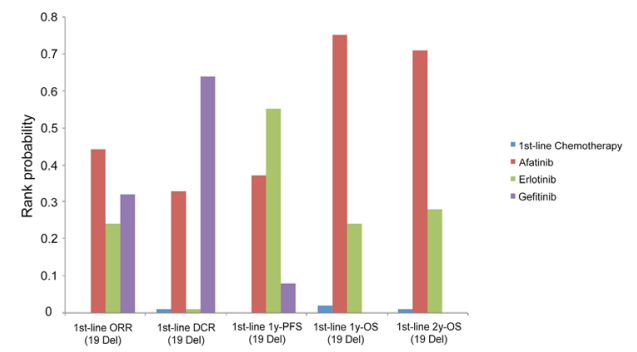

J
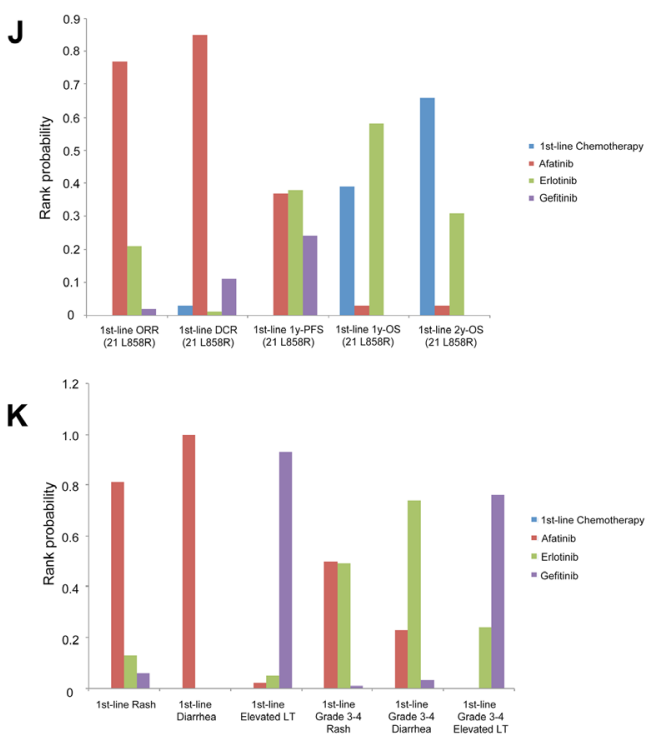

Figure 3: Distribution of probabilities of each agent being ranked the first place based on network $A$ and network $B$. A, B, C, D, E, F were classified by drugs; G, H, I, J, K, L were classified by outcomes. Abbreviations:19 Del, exon 19 deletion; 21 L858R, exon 21 L858R mutation; ORR, objective response rate; DCR, disease control rate; PFS, progression-free survival; OS, overall survival; LT, liver transaminase. 
than gefitinib and higher DCR than erlotinib in EGFR 21 L858R patients. EGFR-TKI showed numerically greater survival benefit in 19 Del compared with chemotherapy, while it was opposite in 21 L858R (Table 3). Gefitinib was not measured in 19 Del / 21 L858R for OS because of lack of available data. Rank probabilities revealed that EGFR-TKIs ranked best for ORR, DCR and 1y-PFS compared with chemotherapy in 19 Del patients, as well as $21 \mathrm{~L} 858 \mathrm{R}$ patients. For 1y-OS (afatinib 0.75, erlotinib 0.24 ) and 2y-OS (afatinib 0.71, erlotinib 0.28), EGFRTKIs still ranked best in 19 Del patients. Nevertheless, the superiority of EGFR-TKI in OS was reduced in 21 L858R patients for 1y-OS (chemotherapy 0.39, erlotinib $0.58)$. Furthermore, chemotherapy ranked best for $2 \mathrm{y}-\mathrm{OS}$ (0.66) compared with EGFR-TKIs (Figure 3D, 3E, 3I, 3J and Table S5).

\section{Network meta-analyses on toxicity in EGFR mutants}

The occurrence rates of rash, grade 3-4 rash and grade 3-4 diarrhea showed no significant differences among gefitinib, elotinib and afatinib. However, afatinib showed higher risk of diarrhea than gefitinib or erlotinib, while gefitinib had greater risk of elevated LT and grade 3-4 elevated LT compared with afatinib or erlotinib (Table $3)$. Rank probabilities showed that afatinib ranked first for the risk of rash (0.81) and diarrhea (1.00) and shared similar rank (0.50) with erlotinib (0.49) for the risk of grade 3-4 rash. Erlotinib ranked first for grade 3-4 diarrhea (0.74) compared with gefitinib and afatinib. Besides, gefitinib ranked first for the risk of elevated LT (0.93) and grade 3-4 elevated LT (0.76) compared with afatinib or erlotinib (Figure 3F, 3K and Table S5).

\section{DISCUSSION}

\section{The origin of this network meta-analysis}

A previous MTC tried to provide indirect comparison for available EGFR-TKIs in treating patients with advanced NSCLC who harbor EGFR mutations. [22] However, at that time, there was no direct head to head comparison between these agents. As a result, the entire network in previous meta-analysis was not wellestablished. Besides, the results of the previous study were short of stratifications by treatment lines or subtypes of EGFR mutations, which didn't reach to precise medical level. Therefore, we still need a large-scale network meta-analysis making multiple comparisons of currently available EGFR-TKIs urgently which integrates the latest data of head to head trials and performs sub-network metaanalyses by different treatment lines and EGFR mutant subtypes.

\section{Intellectual consideration of the established network}

In order to ensure the reliability of research and the exchangeability of cross-treatment, only phase III randomized trials with strict patient allocation and optimized balance between treatment and control group were included in our study. Additionally, icotinib was not enrolled in the network because of following reasons [23]: (i) ICOGEN was the only phase III trial which focused on icotinib by far. It might break the stability of the network because each regimen of the network is encouraged to be linked with other treatments as much as possible. (ii) We still lack of sufficient data on the efficacy of icotinib compared with other EGFR-TKIs or chemotherapy. (iii) Icotinib was not approved in the international market except for China. The world-wide usage rate of icotinib was much lower than gefitinib or erlotinib. Besides, each regimen of the first-line or after first-line chemotherapy was platinum-based doublet or single agent chemotherapy, thus insuring concordant therapeutic efficacy within groups. Therefore, the consistency across the newlyestablished network would harmonize with real situation.

\section{MTC of efficacy in the overall, chemo-naïve and previously treated EGFR mutants}

Our study showed that different EGFR-TKIs shared equivalent curative effect in terms of all outcome measures among the overall, chemo-naïve and previously treated EGFR mutants. EGFR-TKIs were better than firstline chemotherapy in terms of ORR, DCR and 1y-PFS rate, instead of OS. The superiority of EGFR-TKIs in ORR, DCR and PFS for EGFR mutants was due to block of EGFR-driven signals, while the failure to make a distinction for OS between EGFR-TKIs and chemotherapy could be explained by the influence of subsequent crossed treatments.

Rank probabilities showed that erlotinib and afatinib had potentially better efficacy compared with gefitinib in both of the overall and chemo-naïve EGFR mutants. Potentially survival benefit of erlotinib was also observed in previously treated patients compared with gefitinib. Previous trials showed that the reference dose of gefitinib (250 mg qd) was administered at approximately one third of its maximum-tolerated dose (MTD) while erlotinib (150 mg qd) and afatinib (40 mg qd) almost reached their MTDs, respectively. [24-26] Moreover, the halfmaximal inhibitory concentration value of erlotinib was significant smaller than that of gefitinib. [27] As a result, the differences in biological dose of gefitinb, erlotinib and afatinib might be a possible reason for the above trends. Besides, afatinib, as a second-generation TKI, had the ability of irreversibly inhibiting EGFR-kinases and 
suppressing all ErbB receptor family,[28] which might show stronger efficacy than first-generation TKI due to its better binding strength with the EGFR and wider blockade of other signaling networks.

Based on the rank results, afatinib and erlotinib might be superior choices for chemo-naïve EGFR mutant patients as regards efficacy, while erlotinib showed its potentially survival benefit in previously treated patients. However, we still lack of the data of $\geq 2$ nd-line phase III trials on afatinib's efficacy. Erlotinib might be the standard control for trials focused on subsequent treatment after failure of chemotherapy in EGFR mutants.

\section{MTC of efficacy in patients with EGFR $19 \mathrm{Del} /$ 21 L858R}

According to MTC, 19 Del and 21 L858R got numerically greater survival benefit by TKI and chemotherapy, respectively. Moreover, rank probabilities also implied that the superiority of EGFR-TKI in OS in 19 Del patients could not be repeated in 21 L858R patients. Furthermore, chemotherapy revealed greater probability for better efficacy with regards to long-term survival compared with afatinib and erlotinib in 21 L858R patients. As a result, it confirmed that 19 Del was a distinct disease compared with 21 L858R. A well-designed clinical trial of chemotherapy vs. EGFR-TKI in 21 L858R patients should be performed separately in the future. Moreover, scientists should pay attention to the heterogeneity of genetic backgrounds between 19 Del and 21 L858R, which might give the original interpretation of the difference of the curative effect.

\section{MTC of adverse effects in EGFR mutants}

MTC of toxicities showed that each TKI had its own merits and demerits. Afatinib, erlotinib and gefitinib had high, moderate and low risk of rash and diarrhea, respectively, while the occurrence of elevated LT was more common in gefitinib. Recommended EGFR-TKI should be suggested according to patients' tolerability and therapeutic efficacy in clinical practice. It is still unknown whether it is consistent between 19 Del and 21 L858R for dominant adverse effects of different TKIs. More efforts were encouraged to explore the above question in the future.

\section{Potential limitations}

Nevertheless, there exist several limitations. Firstly, data of clinical outcomes were not available in some included studies which might influence statistically significances in comparison analyses. Secondly, we could not exclude the data of few non-classical EGFR mutants in the overall population which might have effects on the efficacy and toxicity of TKIs and cause potential bias.
Future studies were warranted to further testify our results by replenishing unavailable data. MTC by adding the third generation EGFR-TKI (AZD9291 and CO1686) will be expected in a few years.

\section{Conclusion}

Our study indicated a high efficacy-high toxicity pattern of afatinib, a high efficacy-moderate toxicity pattern of erlotinib and a medium efficacy-moderate toxicity pattern of gefitinib. Recommended EGFR-TKI should be suggested according to patients' tolerability and therapeutic efficacy in clinical practice. Moreover, the treatment for advanced EGFR-positive NSCLC might be different between 19 Del and 21 L858R.

\section{MATERIALS AND METHODS}

\section{Study eligibility and identification}

PubMed, Embase, Cochrane Central Register of Controlled Trials were searched respectively to find relevant articles using a combination of the terms "EGFR", "mutation" "19 Del", "21 L858R", "Lung", "NSCLC", "TKI", "gefitinib", "erlotinib" and "afatinib". We also reviewed abstract books and presentations of major recent meetings of ASCO, ESMO, ESMO-Asia and WCLC up to Dec. 2015 to ensure the latest research progress enrolled. Besides, all of the supplemental materials data from each trial were checked and extracted. The literature retrieval was carried out by three reviewers independently. Studies were included if they met the following criteria: (i) phase III randomized trials which reported advanced NSCLC EGFR mutants using specific EGFR-TKI treatment vs. chemotherapy or EGFR-TKI vs. another EGFR-TKI; (ii) trials might be performed in chemo-naïve patients or previously treated patients; (iii) EGFR-TKIs should be gefitinib, erlotinib or afatinib; (iv) EGFR-TKIs were not used as combined therapy or maintenance therapy; (v) at least one clinical outcome was available. Studies failing to meet the above inclusion criteria will be excluded from the network meta-analysis.

\section{Outcomes measures, data extraction and quality assessment}

Therapeutic efficacy and toxicity were clinical outcomes including objective response rate (ORR), disease control rate (DCR), 1y-PFS rate, $1 \mathrm{y}$-OS rate, $2 \mathrm{y}$ OS rate and the rate of rash (grade 3-4 rash), diarrhea (grade 3-4 diarrhea), elevated liver transaminase (LT) (grade 3-4 elevated LT). The data on trial name, patient category, race, therapeutic regimens, EGFR mutation type and above clinical outcomes were extracted by two investigators independently. Two reviewers used the JADAD score to assess the quality of all included studies. 
[29] Discrepancies were discussed by all investigators to reach a consensus. All eligible studies were of high quality after the assessment.

\section{Statistical analyses}

We conducted single-arm meta-analyses with a random effects model to synthesize rates of all the clinical outcomes in EGFR mutants, 19 Del patients and 21 L858R patients stratified by different EGFR-TKI treatments. The results were reported as pooled rates with the corresponding $95 \%$ confidence interval (CI). Statistical heterogeneity across studies was assessed with a forest plot and the inconsistency statistic $\left(\mathrm{I}^{2}\right)$. All calculations were performed using R software, version 2.13.1.

After that, we built a random-effects network within a Bayesian framework using Markov chain Monte Carlo methods in ADDIS 1.15. [30, 31] We networked binary clinical outcomes within studies and specified the relations among the odds ratios (ORs) across studies to make comparisons of different treatments in EGFR mutants, 19 Del patients and 21 L858R patients as previously described. [17] P values less than 0.05 and 95\% CIs were used to assess significance.

Moreover, the probability of the best regimen of each treatment in terms of efficacy and toxicity was also estimated by calculating the OR for each drug compared with an arbitrary common control group, and counting the proportion of iterations of the Markov chain in which each drug had the highest OR, the second highest, and so on. We also ranked the probability to be the best treatment among all the treatment regimens. Agents with greater value in the histogram were associated with greater probabilities for better efficacy or worse toxicity. The inconsistency within the network meta-analysis was evaluated by a variance calculation and a node-splitting analysis as previously reported. [31]

\section{ACKNOWLEDGMENTS}

This study was supported by no fund.

\section{CONFLICTS OF INTEREST}

The authors have declared no conflicts of interest.

\section{Author Contributions}

Conception and design: Yaxiong Zhang, Jin Sheng, Yunpeng Yang, Li Zhang

Literature retrieval: Yaxiong Zhang, Wenfeng Fang, Shiyang Kang

Data extraction and quality assessment: Yang He, Jianhua Zhan, Yuanyuan Zhao, Cong Xue

Statistical guidance: Yaxiong Zhang, Jin Sheng, Shaodong Hong
Data analysis and interpretation: Yuxiang Ma, Ting Zhou, Shuxiang Ma, Suning Ping, Fangfang Gao, Tao Qin, Zhihuang Hu, Ying Tian, Xue Hou, Yan Huang, Ningning Zhou, Hongyun Zhao

Manuscript writing: Yaxiong Zhang, Jin Sheng, Yunpeng Yang, Li Zhang

Primary revision before submitting: All authors

Final approval of manuscript: All authors

\begin{abstract}
Abbreviation
EGFR-TKI epidermal growth factor receptortyrosine kinase inhibitor; NSCLC non-small cell lung cancer; 19 Del EGFR exon 19 deletions; 21 L858R EGFR L858R substitution in exon 21; PFS progressionfree survival; OS overall survival; RCT randomized controlled trial; MTC multiple treatment comparisons; MTD maximum-tolerated dose; ORR objective response rate; DCR disease control rate; LT liver transaminase; OR odds ratio
\end{abstract}

\section{REFERENCES}

1. Maemondo M, Inoue A, Kobayashi K, Sugawara S, Oizumi S, Isobe H, Gemma A, Harada M, Yoshizawa H, Kinoshita I, Fujita Y, Okinaga S, Hirano H, Yoshimori K, Harada T, Ogura T, et al. Gefitinib or chemotherapy for non-small-cell lung cancer with mutated EGFR. The New England journal of medicine. 2010; 362:2380-2388.

2. Mitsudomi T, Morita S, Yatabe Y, Negoro S, Okamoto I, Tsurutani J, Seto T, Satouchi M, Tada H, Hirashima T, Asami K, Katakami N, Takada M, Yoshioka H, Shibata K, Kudoh S, et al. Gefitinib versus cisplatin plus docetaxel in patients with non-small-cell lung cancer harbouring mutations of the epidermal growth factor receptor (WJTOG3405): an open label, randomised phase 3 trial. The Lancet Oncology. 2010; 11:121-128.

3. Fukuoka M, Wu YL, Thongprasert S, Sunpaweravong P, Leong SS, Sriuranpong V, Chao TY, Nakagawa K, Chu DT, Saijo N, Duffield EL, Rukazenkov Y, Speake G, Jiang $\mathrm{H}$, Armour AA, To KF, et al. Biomarker analyses and final overall survival results from a phase III, randomized, open-label, first-line study of gefitinib versus carboplatin/ paclitaxel in clinically selected patients with advanced nonsmall-cell lung cancer in Asia (IPASS). Journal of clinical oncology. 2011; 29:2866-2874.

4. Zhou C, Wu YL, Chen G, Feng J, Liu XQ, Wang C, Zhang S, Wang J, Zhou S, Ren S, Lu S, Zhang L, Hu C, Hu C, Luo $\mathrm{Y}$, Chen L, et al. Erlotinib versus chemotherapy as firstline treatment for patients with advanced EGFR mutationpositive non-small-cell lung cancer (OPTIMAL, CTONG0802): a multicentre, open-label, randomised, phase 3 study. The Lancet Oncology. 2011; 12:735-742.

5. Han JY, Park K, Kim SW, Lee DH, Kim HY, Kim HT, Ahn MJ, Yun T, Ahn JS, Suh C, Lee JS, Yoon SJ, Han JH, Lee JW, Jo SJ, Lee JS. First-SIGNAL: first-line 
single-agent iressa versus gemcitabine and cisplatin trial in never-smokers with adenocarcinoma of the lung. Journal of clinical oncology. 2012; 30:1122-1128.

6. Rosell R, Carcereny E, Gervais R, Vergnenegre A, Massuti B, Felip E, Palmero R, Garcia-Gomez R, Pallares C, Sanchez JM, Porta R, Cobo M, Garrido P, Longo F, Moran $\mathrm{T}$, Insa A, et al. Erlotinib versus standard chemotherapy as first-line treatment for European patients with advanced EGFR mutation-positive non-small-cell lung cancer (EURTAC): a multicentre, open-label, randomised phase 3 trial. The Lancet Oncology. 2012; 13:239-246.

7. Sequist LV, Yang JC, Yamamoto N, O'Byrne K, Hirsh V, Mok T, Geater SL, Orlov S, Tsai CM, Boyer M, Su WC, Bennouna J, Kato T, Gorbunova V, Lee KH, Shah R, et al. Phase III study of afatinib or cisplatin plus pemetrexed in patients with metastatic lung adenocarcinoma with EGFR mutations. Journal of clinical oncology. 2013; 31:3327-3334.

8. Wu YL, Zhou C, Hu CP, Feng J, Lu S, Huang Y, Li W, Hou M, Shi JH, Lee KY, Xu CR, Massey D, Kim M, Shi $\mathrm{Y}$, Geater SL. Afatinib versus cisplatin plus gemcitabine for first-line treatment of Asian patients with advanced non-small-cell lung cancer harbouring EGFR mutations (LUX-Lung 6): an open-label, randomised phase 3 trial. The Lancet Oncology. 2014; 15:213-222.

9. Wu YL, Zhou C, Liam CK, Wu G, Liu X, Zhong Z, Lu S, Cheng Y, Han B, Chen L, Huang C, Qin S, Zhu Y, Pan H, Liang H, Li E, et al. First-line erlotinib versus gemcitabine/ cisplatin in patients with advanced EGFR mutation-positive non-small-cell lung cancer: analyses from the phase III, randomized, open-label, ENSURE study. Annals of oncology. 2015; 26:1883-1889.

10. Yang JC-H, Wu Y-L, Schuler M, Sebastian M, Popat S, Yamamoto N, Zhou C, Hu C-P, O'Byrne K, Feng J, Lu S, Huang Y, Geater SL, Lee KY, Tsai C-M, Gorbunova V, et al. Afatinib versus cisplatin-based chemotherapy for EGFR mutation-positive lung adenocarcinoma (LUX-Lung 3 and LUX-Lung 6): analysis of overall survival data from two randomised, phase 3 trials. The Lancet Oncology. 2015; 16:141-151.

11. Wang H, Huang J, Yu X, Han S, Yan X, Sun S, Zhu X. Different efficacy of EGFR tyrosine kinase inhibitors and prognosis in patients with subtypes of EGFR-mutated advanced non-small cell lung cancer: a meta-analysis. Journal of cancer research and clinical oncology. 2014; 140:1901-1909.

12. Zhang Y, Sheng J, Kang S, Fang W, Yan Y, Hu Z, Hong $\mathrm{S}$, Wu X, Qin T, Liang W, Zhang L. Patients with exon 19 deletion were associated with longer progression-free survival compared to those with L858R mutation after firstline EGFR-TKIs for advanced non-small cell lung cancer: a meta-analysis. PloS one. 2014; 9:e107161.

13. Zhang Y, He D, Fang W, Kang S, Chen G, Hong S, Sheng J, Zhan J, Chen N, Hu Z, Xue C, Yang Y, Ma Y, Qin T, Zhou T, Huang Y, et al. The Difference of Clinical
Characteristics Between Patients With Exon 19 Deletion and Those With L858R Mutation in Nonsmall Cell Lung Cancer. Medicine. 2015; 94:e1949.

14. A. Nishiyama NK, S. Morita, T. Seto, Y. Iwamoto, T. Hirashima, H. Kaneda, T. Kawaguchi, H. Matsuoka, S. Yokota, T. Nishimura, M. Okada, M. Fujita, K. Shibata, Y. Urata, N. Yamamoto, K. Nakagawa, Y. Nakanishi. 1271PRANDOMIZED PHASE III STUDY COMPARING GEFITINIB (G) WITH ERLOTINIB (E) IN PATIENTS (PTS) WITH PREVIOUSLY TREATED ADVANCED LUNG ADENOCARCINOMA (LA): WJOG 5108L. Annals of oncology. 2014; 25:iv426-iv470.

15. Jin-ji Yang QZ, Hong-Hong Yan, Xu-Chao Zhang, Hua-Jun Chen, Han-Yan Tu, et al. A Randomized Controlled Trial of Erlotinib versus Gefitinib in Advanced Non-Small-Cell Lung Cancer Harboring EGFR Mutations (CTONG0901). Journal of thoracic oncology. 2015; 10.

16. Keunchil Park E-HT, Li Zhang, Vera Hirsh, Kenneth O’Byrne, Michael Boyer, James Chih-Hsin Yang, Tony Mok, Miyoung Kim, Dan Massey, Victoria Zazulina, Luis Paz-Ares. Afatinib versus gefitinib as first-line treatment for patients with advanced non-small cell lung cancer harboring activating EGFR mutations: results of the global, randomized, open-label, Phase IIb trial LUX-Lung 7. Annals of oncology. 2015.

17. Cipriani A, Furukawa TA, Salanti G, Geddes JR, Higgins JP, Churchill R, Watanabe N, Nakagawa A, Omori IM, McGuire H, Tansella M, Barbui C. Comparative efficacy and acceptability of 12 new-generation antidepressants: a multiple-treatments meta-analysis. Lancet (London, England). 2009; 373:746-758.

18. Maruyama R, Nishiwaki Y, Tamura T, Yamamoto N, Tsuboi M, Nakagawa K, Shinkai T, Negoro S, Imamura F, Eguchi K, Takeda K, Inoue A, Tomii K, Harada M, Masuda N, Jiang H, et al. Phase III study, V-15-32, of gefitinib versus docetaxel in previously treated Japanese patients with non-small-cell lung cancer. Journal of clinical oncology. 2008; 26:4244-4252.

19. Douillard JY, Shepherd FA, Hirsh V, Mok T, Socinski MA, Gervais R, Liao ML, Bischoff H, Reck M, Sellers MV, Watkins CL, Speake G, Armour AA, Kim ES. Molecular predictors of outcome with gefitinib and docetaxel in previously treated non-small-cell lung cancer: data from the randomized phase III INTEREST trial. Journal of clinical oncology. 2010; 28:744-752.

20. Ciuleanu T, Stelmakh L, Cicenas S, Miliauskas S, Grigorescu AC, Hillenbach C, Johannsdottir HK, Klughammer B, Gonzalez EE. Efficacy and safety of erlotinib versus chemotherapy in second-line treatment of patients with advanced, non-small-cell lung cancer with poor prognosis (TITAN): a randomised multicentre, open-label, phase 3 study. The Lancet Oncology. 2012; 13:300-308.

21. Kawaguchi T, Ando M, Asami K, Okano Y, Fukuda M, Nakagawa H, Ibata H, Kozuki T, Endo T, Tamura A, 
Kamimura M, Sakamoto K, Yoshimi M, Soejima Y, Tomizawa $\mathrm{Y}$, Isa $\mathrm{S}$, et al. Randomized phase III trial of erlotinib versus docetaxel as second- or third-line therapy in patients with advanced non-small-cell lung cancer: Docetaxel and Erlotinib Lung Cancer Trial (DELTA). Journal of clinical oncology. 2014; 32:1902-1908.

22. Liang $\mathrm{W}, \mathrm{Wu} \mathrm{X}$, Fang $\mathrm{W}$, Zhao Y, Yang Y, Hu Z, Xue C, Zhang J, Zhang J, Ma Y, Zhou T, Yan Y, Hou X, Qin $\mathrm{T}$, Dinglin $\mathrm{X}$, Tian $\mathrm{Y}$, et al. Network meta-analysis of erlotinib, gefitinib, afatinib and icotinib in patients with advanced non-small-cell lung cancer harboring EGFR mutations. PloS one. 2014; 9:e85245.

23. Shi Y, Zhang L, Liu X, Zhou C, Zhang L, Zhang S, Wang D, Li Q, Qin S, Hu C, Zhang Y, Chen J, Cheng Y, Feng $\mathrm{J}$, Zhang $\mathrm{H}$, Song $\mathrm{Y}$, et al. Icotinib versus gefitinib in previously treated advanced non-small-cell lung cancer (ICOGEN): a randomised, double-blind phase 3 noninferiority trial. The Lancet Oncology. 2013; 14:953-961.

24. Hidalgo M, Siu LL, Nemunaitis J, Rizzo J, Hammond LA, Takimoto C, Eckhardt SG, Tolcher A, Britten CD, Denis L, Ferrante K, Von Hoff DD, Silberman S, Rowinsky EK. Phase I and pharmacologic study of OSI-774, an epidermal growth factor receptor tyrosine kinase inhibitor, in patients with advanced solid malignancies. Journal of clinical oncology. 2001; 19:3267-3279.

25. Baselga J, Rischin D, Ranson M, Calvert H, Raymond E, Kieback DG, Kaye SB, Gianni L, Harris A, Bjork T, Averbuch SD, Feyereislova A, Swaisland H, Rojo F, Albanell J. Phase I safety, pharmacokinetic, and pharmacodynamic trial of ZD1839, a selective oral epidermal growth factor receptor tyrosine kinase inhibitor, in patients with five selected solid tumor types. Journal of clinical oncology. 2002; 20:4292-4302.
26. Gordon MS, Mendelson DS, Gross M, Uttenreuther-Fischer M, Ould-Kaci M, Zhao Y, Stopfer P, Agus DB. A Phase I, open-label, dose-escalation study of continuous once-daily oral treatment with afatinib in patients with advanced solid tumors. Investigational new drugs. 2013; 31:409-416.

27. Zhao Q, Shentu J, Xu N, Zhou J, Yang G, Yao Y, Tan F, Liu D, Wang Y, Zhou J. Phase I study of icotinib hydrochloride (BPI-2009H), an oral EGFR tyrosine kinase inhibitor, in patients with advanced NSCLC and other solid tumors. Lung cancer. 2011; 73:195-202.

28. Yang JC, Shih JY, Su WC, Hsia TC, Tsai CM, Ou SH, Yu CJ, Chang GC, Ho CL, Sequist LV, Dudek AZ, Shahidi M, Cong XJ, Lorence RM, Yang PC, Miller VA. Afatinib for patients with lung adenocarcinoma and epidermal growth factor receptor mutations (LUX-Lung 2): a phase 2 trial. The Lancet Oncology. 2012; 13:539-548.

29. Moher D, Jadad AR, Nichol G, Penman M, Tugwell P, Walsh S. Assessing the quality of randomized controlled trials: an annotated bibliography of scales and checklists. Controlled clinical trials. 1995; 16:62-73.

30. Salanti G, Higgins JP, Ades AE, Ioannidis JP. Evaluation of networks of randomized trials. Statistical methods in medical research. 2008; 17:279-301.

31. Valkenhoef GV, Tervonen T, Zwinkels T, Brock BD, Hillege H. ADDIS: A decision support system for evidence-based medicine. ERIM Top-Core Articles. 2012; 55:459-475. 